\title{
Reduction of Sphero-Chromatic Aberration in Catadioptric Systems
}

\author{
By Robert E. Stephens
}

\begin{abstract}
Schmidt and Maksutov catadioptric systems suffer from sphero-chromatic aberrations that are of opposite sign. By the use of a hybrid system, whose corrector is a deep-meniscus lens with an aspheric curve on the external surface, the sphero-chromatic residuals have been reduced and, as a consequence, usuable relative apertures have been increased.
\end{abstract}

Some time ago a Maksutov camera system of large relative aperture was designed at the National Bureau of Standards for the purpose of ascertaining whether or not such a system, employing as it does only spherical surfaces, could be used to replace a Schmidt system for certain purposes. It was found that the residual zonal spherical aberration was so large that the system was unsatisfactory. On further analysis, however, it was found that the residual sphero-chromatic aberration of the Maksutov system was opposite in sign to that of a comparable Schmidt system and of about the same magnitude. This suggested that a hybrid system, partially corrected by an aspheric surface and partially by a deep meniscus lens, would suffer substantially less from sphero-chromatic aberration than either the Schmidt or the Maksutov type. Such has indeed been found to be the case.

A hybrid Maksutov-Schmidt system consisting of a correcting plate, plane on one side, placed with its vertex at the center of the concave mirror, and a meniscus achromatic lens properly placed, could presumably be designed that would be as free from coma as the best of either of the original types. This has not been attempted by the author. Instead, several systems have been designed in which the aspheric surface is applied to the side of the meniscus lens away from the mirror.

The chromatic aberration of either a Schmidt or a Maksutov system results from the variation of correction for spherical aberration with index

1 This work has been sponsored by the Bureau of Ships, Navy Department. of refraction of the correcting plate or meniscus lens. In either of these systems the variation of spherical correction is preponderantly due to changes of the third order spherical aberration of the correcting plate or lens. In a Schmidt system the speherical aberration is perfectly corrected for one wavelength. At other wavelengths there exists simple spherical aberration, undercorrected for longer wavelengths and overcorrected for shorter wavelengths. In a Maksutov system the situation is reversed. Superimposed upon the zonal spherical aberration, which is the best that can be accomplished by the meniscus lens, is overcorrection of spherical aberration at longer wavelengths and under correction at shorter wavelengths. Because of the large angles of incidence existing at the surfaces of the lens in this type system, aberrations of higher order are present to a greater extent than in the Schmidt system, but the differences over a small range of wavelengths are still predominantly of the third order.

For the first trial it was intended to design a system with a relative aperture of $f / 0.7$, which would be achromatic at $f / 1.0$. However, because of the effect of the corrector upon the equivalent focal length, the achromatic zone turned out to be at $f / 0.89$. A first radius and a suitable thickness were assumed for the corrector. The second radius was then given the value that resulted in achromatism at the selected zone. Rays were then traced through the system at the most important wave length and the condition of the spherical aberration determined. Different values were assumed for the first radius until a system 
of the Maksutov type was found in which the marginal longitudinal spherical aberration was about half that for the uncorrected mirror.

The first surface was then replaced by an aspheric surface ${ }^{2}$ such that the optical path lengths for the principal wavelength from all points of an incident plane wave to the chosen focus were made equal. The chosen focus was that corresponding to the ray of the principal wavelength through the achromatic zone of the tentative spherical surface. The resulting aspheric surface is tangent to the tentative surface at the achromatic zone.

The system was then analyzed for residual chromatic aberration by tracing rays at the extreme wavelengths through the system. The longitudinal displacements, from the focus of the rays of the most important wavelength, are shown graphically as a function of height of incidence in figure 2. This should be compared with a similar graph for a Schmidt system of somewhat smaller relative aperture $(f / 0.83)$ shown in figure 1 . The improvement is obviously considerable in spite of the larger relative aperture of the hybrid system.

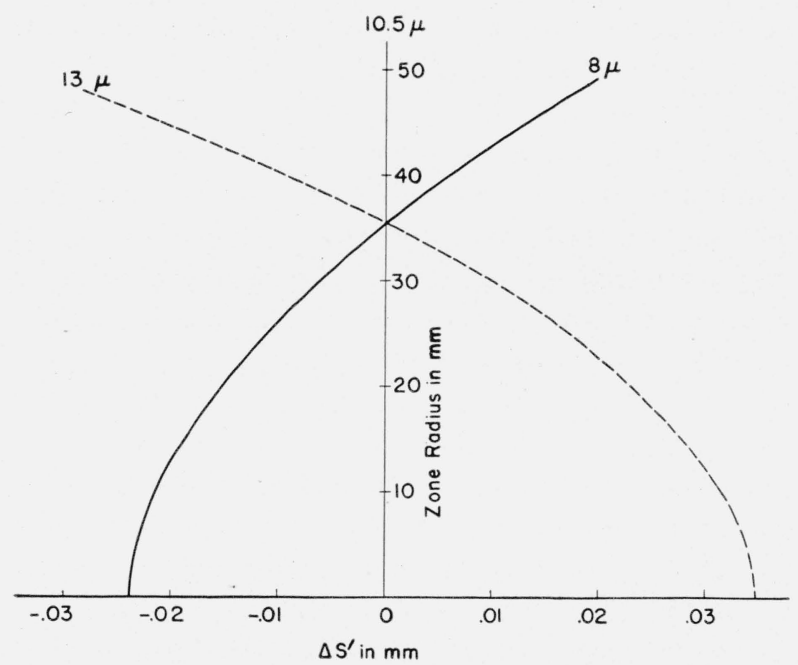

Figure 1. Spherical aberration curves for a Schmidt camera system.

Equivalent focal length $=83.56 \mathrm{~mm}$.

Although the improvement over previous systems is considerable, the balance of the residual spherochromatic aberration is not optimum. This optimum condition would be attained if the

\footnotetext{
${ }^{2}$ It is not the purpose of this paper to describe the methods used to com pute aspheric surfaces. The procedures used were similar to the exact method of F. A. Lucy, J Opt. Soc. Am. 30, 251 (1940); and, for finite object distances, H. S. Friedman, J. Opt. Soc. Am., 37, 480 (1947).
}

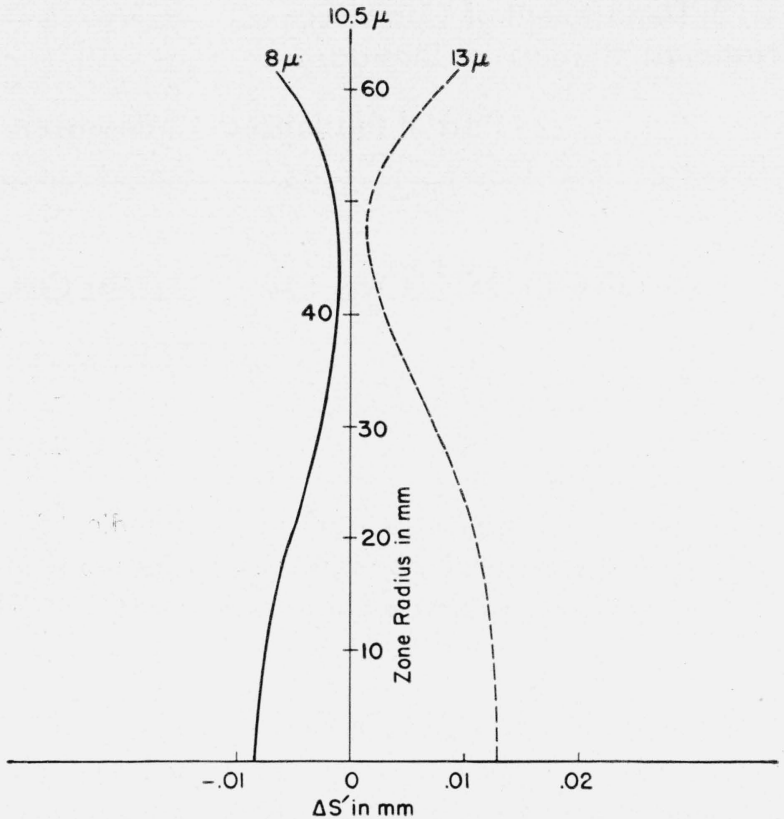

Figure 2. Spherical aberration of first Schmidt-Maksutov hybrid.

Equivalent focal length $=88.68 \mathrm{~mm}$.

best focus (in the presence of residual aberration) for each of the extreme wavelengths could be made to coincide with the perfect focus of the principal wavelength. If this is impossible, the next best adjustment would be such that the focal ranges ${ }^{3}$ for the extreme wavelengths overlap. Accordingly, further trials were made.

In succeeding designs the second radius of the corrector was so chosen that modest overcorrection of chromatic aberration was obtained at the selected zone. Then, when the aspheric surface had been substituted for the tentative first surface, correction of the type illustrated in figure 3 resulted. The residual optical path differences, at the focus of the principal wavelength, were less than one-tenth wavelength for the extreme wavelengths. The coma of the system, as indicated by its maximum offense against the sine theorem of 0.007 , is greater than would usually be tolerated, but was quite satisfactory for the purpose at hand. Figure 4 shows a drawing of this system.

The systems designed by the author were all for use in the infra-red region between $8 \mu$ and $13 \mu$, $10.5 \mu$ being chosen as the principal wavelength.

\footnotetext{
"A discussion of the "focal range" is given in Glazebrook, Dictionary of applied physics, in the section on the opties of the microscope (written by Conrady), IV, 221.
} 


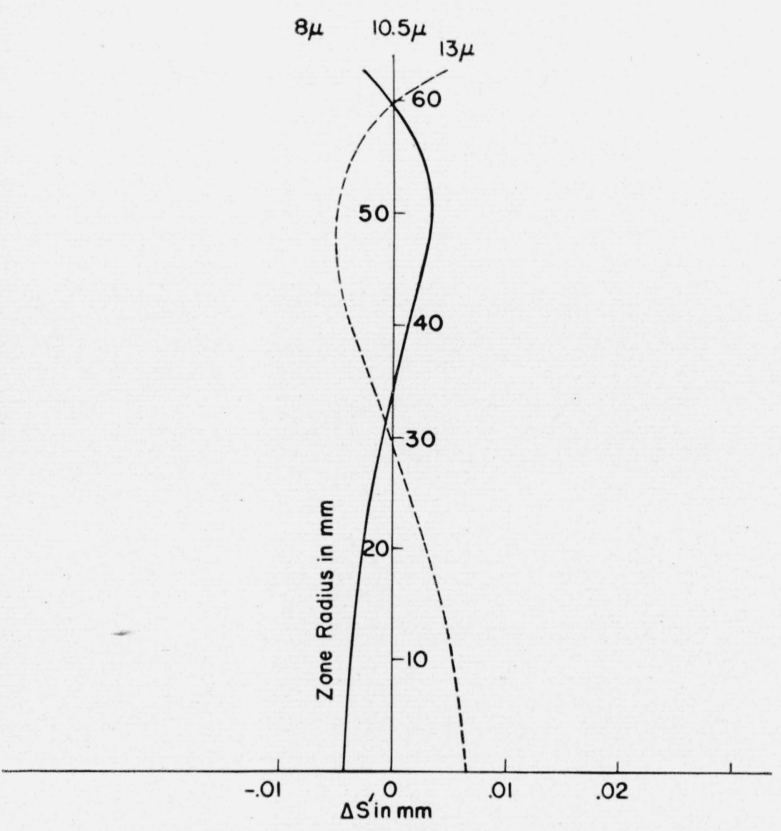

Figure 3. Spherical aberration of Schmidt-Maksutov hybrid in which almost optimum balance of residual spherochromatic aberration has been achieved.

Equivalent focal length $=89.66 \mathrm{~mm}$.

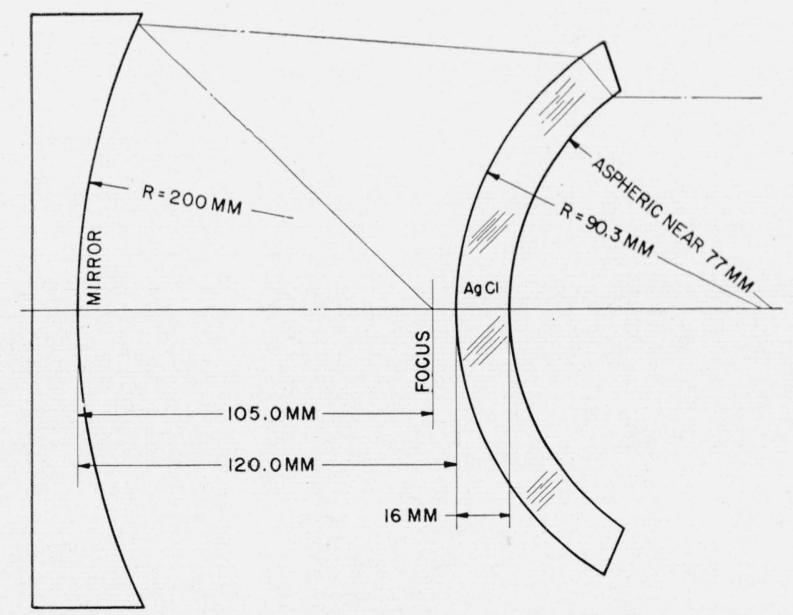

Figure 4. System whose aberrations are shown in figure 3.

The principles involved in the reduction of the residual spherochromatic aberration should, however, apply equally well to systems for use in the visible or any other spectral range.

Washington, December 19, 1948. 